\title{
Experimental Data for the Validation of Numerical Methods - SAE Reference Notchback Model.
}

\author{
Author, co-author (D Wood, M A Passmore, A K Perry) \\ Affiliation (Loughborough University)
}

Copyright @ 2014 SAE International

\begin{abstract}
The use of simulation tools by vehicle manufacturers to design, optimize and validate their vehicles is essential if they are to respond to the demands of their customers, to meet legislative requirements and deliver new vehicles ever more quickly. The use of such tools in the aerodynamics community is already widespread, but they remain some way from replacing physical testing completely. Further advances in simulation capabilities depend on the availability of high quality validation data so that simulation code developers can ensure that they are capturing the physics of the problems in all the important areas of the flow-field.
\end{abstract}

This paper reports on an experimental program to generate such high quality validation data for a SAE 20 degree backlight angle notchback reference model. This geometry is selected as a particularly powerful test case for the development and validation of numerical tools because the flow exhibits a realistic impingement and A pillar regime, significant three dimensional structures and the backlight/boot-deck exhibits a local separation and reattachment. The paper includes force and moment data, surface pressures for the centerline, slant, boot-deck and base and detailed PIV data for the impingement region, model centerline, A pillar and multiple planes on the slant and boot-deck. Time averaged, statistical and instantaneous data are presented.

Results are discussed with regard to the overall flow features, the correlation between the different data sets and the accuracy and limitations of each of the experimental techniques in this particular application. Example data is included throughout the paper and full data sets are freely available in the Loughborough University Institutional Repository as a resource for future code development.

\section{Introduction}

Vehicle manufacturers face considerable challenges in the design, optimization and delivery of their products to meet the needs of their various stakeholders. They must respond to the demands of their customers for ever higher quality and value, they must meet complex and changing legislative requirements and deliver new vehicles increasingly quickly. The use of simulation tools is essential if these demands are to be met and consequently manufacturers and suppliers have invested Page 1 of 10 heavily in simulation capability. The use of simulation tools in the aerodynamics community is already widespread, but they remain some way from replacing physical testing completely. In the aerodynamics community this is clear from the continued development of existing wind tunnel facilities and the construction of new ones.

Simulation capability within aerodynamics has improved over the last several decades but further advances require a shift away from validation using a bottom line drag figure to a much more complete assessment of the performance. For example, it is not uncommon for a numerical prediction to generate a drag coefficient within only one or two percent of an experimental value, but the same prediction to be $30 \%$ or more adrift in the mean base pressure. Computational methods inherently generate a wealth of detailed data that has considerable value in the design and optimization process but the value this offers will only be realized if there is full confidence in the output. High quality validation data generated from experimental work has an important part to play because code developers can use it to demonstrate that they are capturing the physics of the problems in all the important areas of the flow-field, or to identify areas of significant error. The purpose of this paper is to make high quality validation data available to the wider community. Initially this is for a single test geometry that captures the most important aspects of a vehicle flow-field but there is the potential to add to this resource. This paper does not attempt to present any CFD results as the intention is to provide the resource rather than another Experimental/CFD comparison.

The data can be downloaded free of charge from the Loughborough University Institutional Repository [1]. The download includes details of the geometry, full descriptions of data and its location in the model frame of reference.

\section{Experimental methodology}

The approach taken is to use a standard reference model that is already widely available and accepted as a reliable test case for vehicle aerodynamics. The use of simple bodies and simplified vehicle models is well-established within the automotive aerodynamics community because they provide a means of capturing the important flow-field structures and physics without the distraction of specific vehicle details. The 
most resonant examples are those of Morel [2] and Ahmed [3]; these examples considered just one aspect of vehicle aerodynamics, but have proved invaluable since, both in the insight provided into the flow-field and also in generating understanding that is applied to real vehicle development.

LeGood and Garry [4] provide a thorough summary of the use of reference models, including simple bodies, basic car shapes and production series cars.

In this work the SAE 20 degree backlight angle notchback reference model is selected, having been designed as a basic car shape rather than a simple body such as the Ahmed model. It therefore exhibits a realistic impingement and $A$ pillar flow, significant three dimensional structures and the backlight/boot-deck region exhibits a local separation and reattachment; making this geometry a powerful test case for the development and validation of numerical tools.

It is worth noting that the new widely available generic car model, Heft et al, [5] is not considered suitable for this work. The proposed vehicle contains many of the features seen on a typical vehicle while seeking to make a realistic, but nonmanufacturer specific, geometry widely available. The more complex geometry may be useful for broad evaluation purposes but is not justified here because the additional detail and complexity do not add significant new numerical challenges over a more simple geometry, they do however increase computational load and introduce difficulties of interpretation.

The experimental program consists of force and moment measurements, surface pressures for the centerline, slant, boot-deck and base and detailed PIV data for the impingement region, model centerline, A pillar and multiple planes on the slant and boot-deck. Time averaged, statistical and instantaneous data are presented.

\section{Wind tunnel}

All data was acquired in the Loughborough University $1 / 4$ scale wind tunnel illustrated in Figure 1. Additional details including inlet profile and detailed geometry can be found in Johl, [6]. The working section has a $1.92 \mathrm{~m} \times 1.32 \mathrm{~m}$ cross section expanding to $1.94 \mathrm{~m} \times 1.32 \mathrm{~m}$ over its $3.2 \mathrm{~m}$ length to negate the effects of boundary layer growth on axial pressure distribution. The walls and a section of the roof are constructed from toughened glass allowing optical access. The floor is fixed, generating a boundary layer thickness $\left(\delta^{99}\right)$ of $60 \mathrm{~mm}$ at the model location.

Test section working velocity is $40 \mathrm{~m} / \mathrm{s}$, turbulence intensity at this velocity is $0.2 \%$ and the deviation from the bulk flow velocity is $\pm 0.4 \%$. The temperature, free-stream static and dynamic pressure are measured at the start of the working section with a pitot-static tube and thermocouple; all of this data is delivered digitally to the control computer which is used to control tunnel speed and to calculate force and moment coefficients. The atmospheric pressure is measured with a Druck DPI 142 barometer located in the wind tunnel control room.

Page 2 of 10

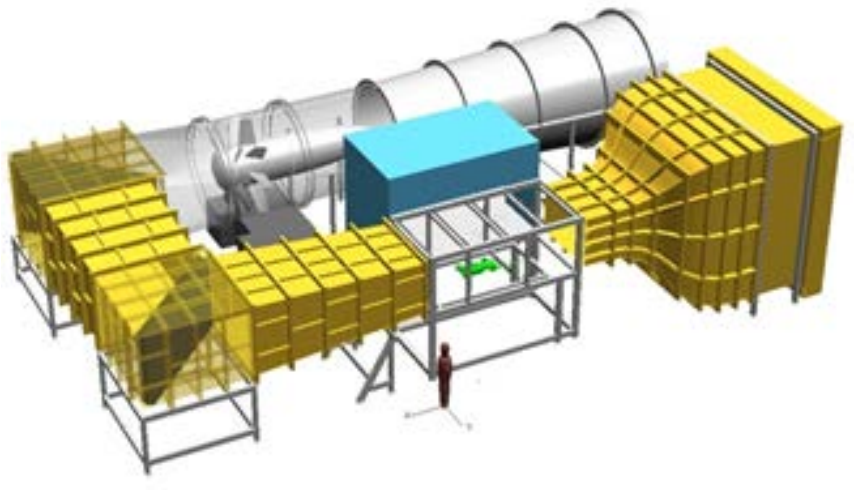

Figure 1 Loughborough University wind tunnel layout.

\section{Model description}

The SAE reference model has been utilized by previous investigations in work such as the development of open jet wind tunnel boundary corrections [7], parametric studies analyzing the effects of ground simulation in wind tunnels [8], experimental work validating CFD simulations [9] and the investigation of dynamic yaw behavior [10]. These have been conducted across a range of scales from 1:5 up to full scale. The 1:5 scale is employed here as it provides a suitable compromise between factors such as wind tunnel blockage, manufacturability, achievable Reynolds number and the field of view that can be reasonably resolved with PIV.

The SAE reference model is an evolution of the MIRA family of reference models and incorporates a number of small but significant improvements. The front end is simplified and formed from a $30^{\circ}$ slant with a large leading edge radius. This feeds a flat floor with a small $6^{\circ}$ centrally mounted diffuser starting at the rear axle centerline designed to counteract the large negative pitching moment generated by the front slant. Surface intersections are blended with large radii minimizing flow separation and providing a degree of similarity with production vehicles, where flow typically remains attached as it transitions between the forward facing and longitudinal surfaces. The longitudinal radii are, however, of a slightly more complex, elliptical design incorporating a sharp edge intended to fix the location of separation over a range of yaw angles and reduce the models Reynolds sensitivity at yaw. The design omits wheels and wheel arches and is instead mounted via 4 slender cylindrical pins. Full details of the model are available in the validation data set available for download. 

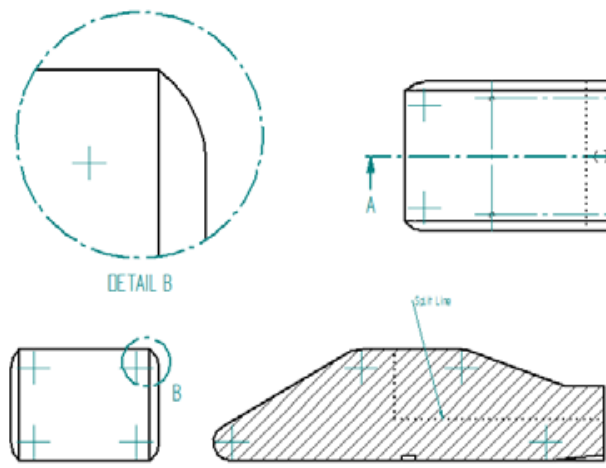

SECTION A-A
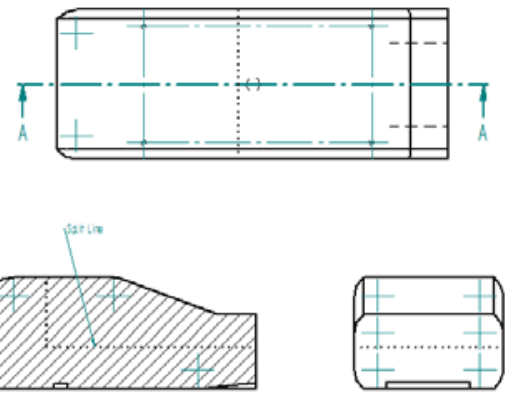

Figure 2 Model geometry - SAE reference model saloon with 20 degree back-slant.

\section{Balance measurements}

Force and moment measurements were made using an underfloor 6 component virtual center balance fitted with an integrated yaw drive system. The model is mounted to the balance via four $8 \mathrm{~mm}$ diameter pins protruding through $10 \mathrm{~mm}$ clearance holes in the tunnel floor. These are located $245 \mathrm{~mm}$ forward and rearward of the model centerline and $35 \mathrm{~mm}$ inboard of the edges. The ranges and accuracies of the forces and moments are contained within table 1 , and refer specifically to the calibration performed when the balance was installed. When testing a $1 / 4$ scale automotive model; a repeatability of $+/-2$ drag counts is typical when fully removing and reinstalling the model. All force and moment data reported in this paper is averaged over a 30 second sampling period.

Table 1. Under-floor balance force and moment accuracy.

\begin{tabular}{|l|l|l|}
\hline Component & $\begin{array}{l}\text { Force and Moment } \\
\text { Ranges }\end{array}$ & $\begin{array}{l}\text { Accuracy (\% of full } \\
\text { scale) }\end{array}$ \\
\hline Drag, $\mathrm{C}_{\mathrm{d}}$ & $\pm 120 \mathrm{~N}$ & 0.010 \\
\hline Side Force $\mathrm{C}_{\mathrm{y}}$ & $\pm 420 \mathrm{~N}$ & 0.005 \\
\hline Lift $\mathrm{C}_{\mathrm{l}}$ & $\pm 500 \mathrm{~N}$ & 0.010 \\
\hline Roll Moment, $\mathrm{C}_{\mathrm{mx}}$ & $\pm 150 \mathrm{Nm}$ & 0.010 \\
\hline Pitching Moment, $\mathrm{C}_{\mathrm{my}}$ & $\pm 60 \mathrm{Nm}$ & 0.010 \\
\hline Yawing Moment, $\mathrm{C}_{\mathrm{mz}}$ & $\pm 45 \mathrm{Nm}$ & 0.015 \\
\hline
\end{tabular}

\section{Pressure measurements}

The model incorporates 110 pressure tappings formed from $25 \mathrm{~mm}$ long, $0.9 \mathrm{~mm}$ I.D. brass tubes fitted flush to the surface enabling a pair of 64 channel scanners to acquire surface pressure remotely via lengths of small bore flexible tubing. The pressure scanners had a manufacturer quoted accuracy of $\pm 1.47 \mathrm{~Pa}$. The pressure tappings are arranged in a grid formation across the backlight, boot-deck and base and are distributed over half the model up to the centerline to increase the spatial resolution afforded by the finite number available. In addition, 30 tappings are distributed around the upper body on the centerline enabling over-body centerline pressure

Page 3 of 10 distribution to be obtained. An illustration of their arrangement is shown in figure 3.

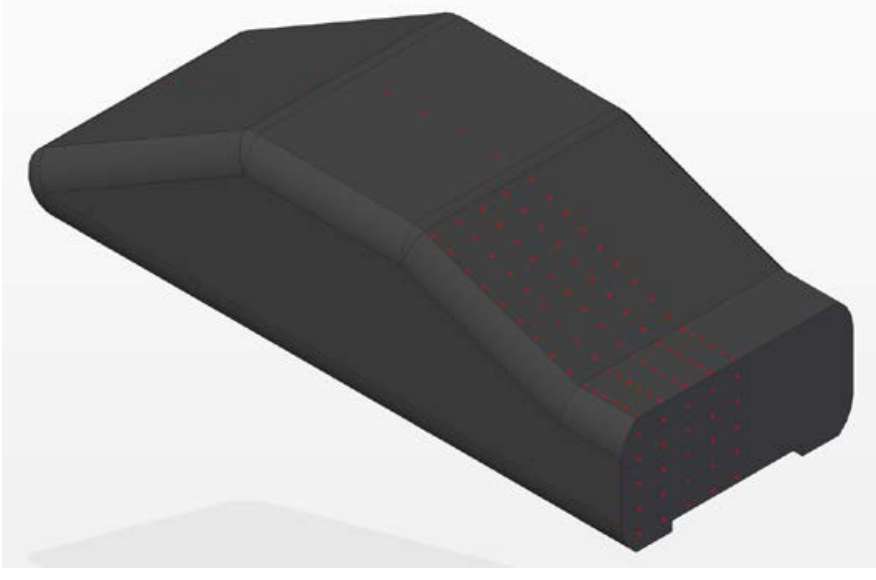

Figure 3 Model pressure tapping distribution.

At each pressure tapping 8192 pressure samples were collected at $260 \mathrm{~Hz}$, representing 31 seconds of data. Pressure signal distortion caused by the tubing linking the model surface to the pressure scanner was removed using an experimentally derived correction function found using a similar method as Sims-Williams and Dominy [11]. The surface pressures are presented as pressure coefficients based on free-stream dynamic pressure and were corrected for blockage using the MIRA blockage correction.

\section{PIV measurements}

Images were collected using two 4 Mega -pixel dual-frame cameras with 14 bit $2048 \times 2048$ pixel CCD sensors. The light sheet was generated using a dual pulse $532 \mathrm{~nm}$ Nd-YAG $200 \mathrm{~mJ}$ laser with the beam conditioned through spherical and cylindrical lenses. The light sheet is approximately $1 \mathrm{~mm}$ thick across the measurement plane. The flow was seeded in both the tunnel contraction and through the floor at the start of the working section with atomized olive oil, generating particles with diameters between 1 and $2 \mu \mathrm{m}$.

The inter-frame time was optimized, for each configuration, such that the resulting images have as close as possible to the ideal average pixel shift of $a^{1 / 4}$ of the final interrogation window size. In most cases the final interrogation size was $32 \times 32$ pixels.

The vector calculation depends on determining the average particle displacement in an interrogation cell through the calculation of the spatial correlation between the first and second frame of a raw image pair. To avoid problems of peak locking (Westerweel [12]) the setup was optimized in each case to ensure a minimum particle diameter, in the recorded image, of 2 pixels. Pre-processing of the images was avoided apart from masking and subtracting an unseeded background image to reduce problems of glare and laser reflection.

In general the processing used a multi-pass approach, initially with a $128 \times 128$ pixel interrogation cell to calculate a reference vector field which was subsequently used to adaptively shift 
the cells as their size was reduced to $32 \times 32$. To increase data yield, interrogation cells were overlapped by $50 \%$, this has the added advantage of improving the validation process,

Westerweel [12]. After the velocity field has been calculated an automated validation process was applied to evaluate data quality and eliminate spurious vectors using a median filter. Where a vector was eliminated it was replaced using the vector identified from the second strongest correlation peak if this met the validation criteria. If not, it was replaced via linear interpolation. Replacing the vector avoids contaminating statistics with zero or missing values.

In all measurement planes reported in this paper the time averaged data is calculated from the average of 1000 instantaneous vector fields. The accuracy of the mean velocity at any point within the flow depends primarily on the local turbulence intensity and hence RMS. A single figure for accuracy is therefore not appropriate. Typically for this type of velocity field with a freestream velocity of $40 \mathrm{~m} / \mathrm{s}$ the accuracy in the mean is of order $\pm 0.2 \%$ in the freestream reducing to approximately $\pm 2 \%$ in the wake. Some examples for similar flows are provided by Passmore [13]. The published data contains the RMS velocity at each vector location allowing a confidence interval to be determined at any point. An illustration of all the PIV measurement planes included in the dataset is shown in Figure 4.

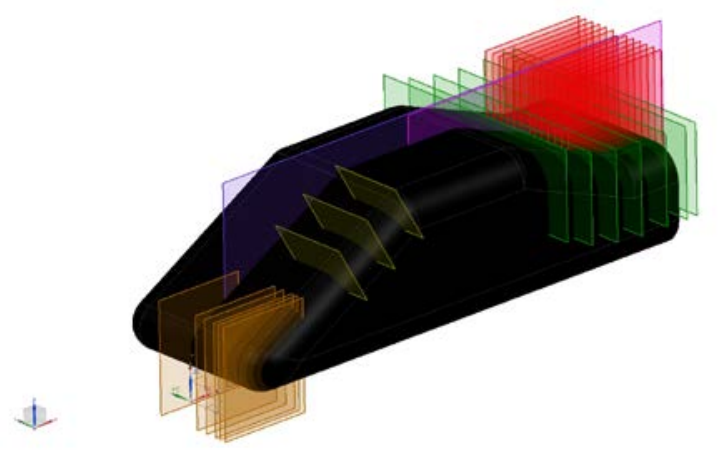

Figure 4 Illustration of all PIV measurement planes.

\section{Results and Discussion}

The coefficients calculated from the balance data are presented in Figure 4, all coefficients have been corrected for blockage using the Mercker blockage correction [14].

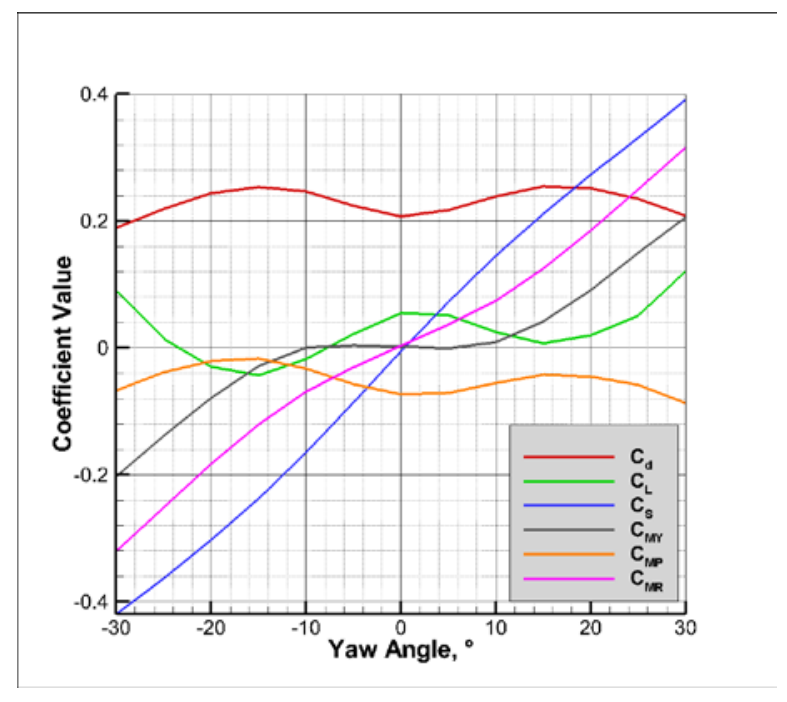

Figure 5 Force and moment coefficients as a function of yaw angle.

\section{Centerline and Impingement region}

The over-body centerline pressure distribution is shown in Figure 5. High static pressure is present immediately downstream of the nose where flow accelerates away from the front stagnation point. This falls to a local minimum at the slant header where the curvature induces high levels of acceleration and velocity. Pressure recovers over the roof before forming a second minimum at the rear slant header where acceleration is again high due to the local curvature. Downstream of this pressure recovers as the flow diffuses and decelerates into the notches expanding cross section. A slight inflection can be seen in the pressure trace toward the top of the slant at the point separation occurs, although pressure recovery continues, albeit at a slightly reduced rate downstream of this point. Pressure subsequently peaks on the boot-deck where the over-body flow reattaches and impinges, partially stagnating, before falling again as it accelerates away toward the bootdeck trailing edge where it finally separates to form the far wake.

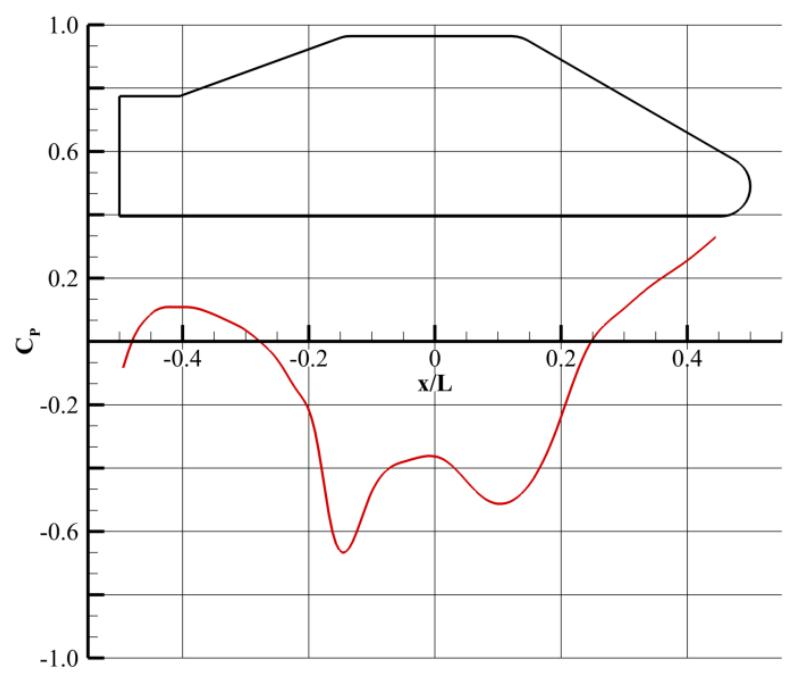

Figure 6 Over-body centerline pressure distribution. 
A complete plane of 2D PIV data was acquired along the centerline using the arrangement shown in Figure 6 to capture an $850 \times 400 \mathrm{~mm}$ field of view. The time-averaged velocity field acquired from this measurement is shown in Figure 7, with streamlines overlaid upon contours of RMS velocity.

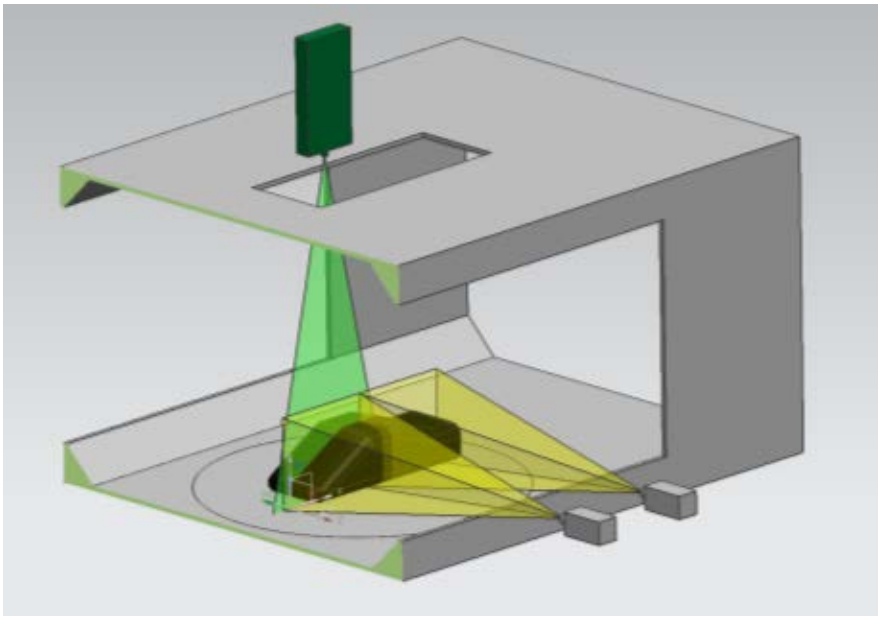

Figure 7. PIV arrangement for complete centerline. Two component large field of view.

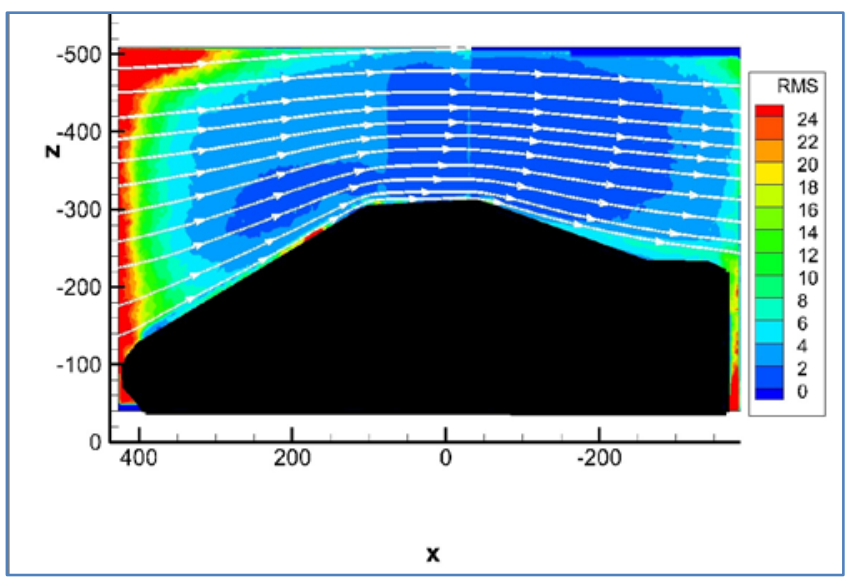

Figure 8 Full centreline PIV. Streamlines with RMS velocity contours.

To provide more detail in the impingement zone the PIV configuration illustrated in Figure 6 was modified to allow stereoscopic measurements to be taken over the front half of the model. Five planes were acquired: on the centerline $(y / w=0.0)$ and at $y / w=-0.25,-0.31,-0.375$ and -0.44 (Where $w$ is the model width). Figure 8 shows the results of these for the centerline and $y / w=-0.44$, presented in the form of in-plane streamlines upon contours of through plane velocity. It is clear that there is some out of plane velocity at the centerline suggesting a slight asymmetry in the flow. At $y / w=-0.44$ this has increased as the flow behaves in a more 3 dimensional manner, accelerating around the edge of the nose and onto the A pillar radius.

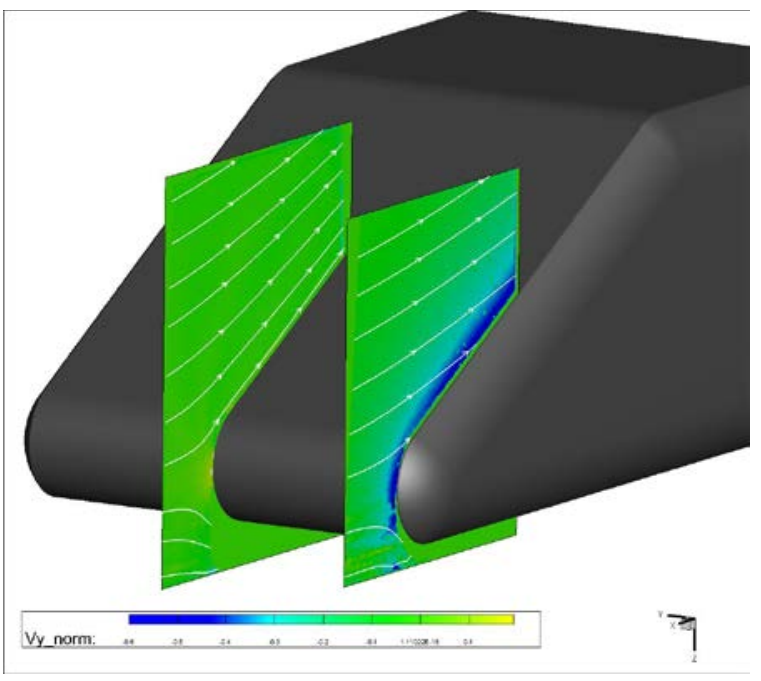

Figure 9 Front impingement centerline $(y / w=0)$ and $y / w=-0.44$. In plane streamlines upon contours of through plane velocity.

\section{A pillar}

2D 2-component PIV measurements have been taken along 3 planes intersecting the A-Pillar at 50\%, $70 \%$ and $90 \%$ of its length and orientated normal to the front slant. This data was acquired at 4 yaw angles $0^{\circ},-10^{\circ},-20^{\circ}$ and $-30^{\circ}$ on the lee side where flow has a greater propensity to separate. The PIV arrangement is illustrated in Figure 9.

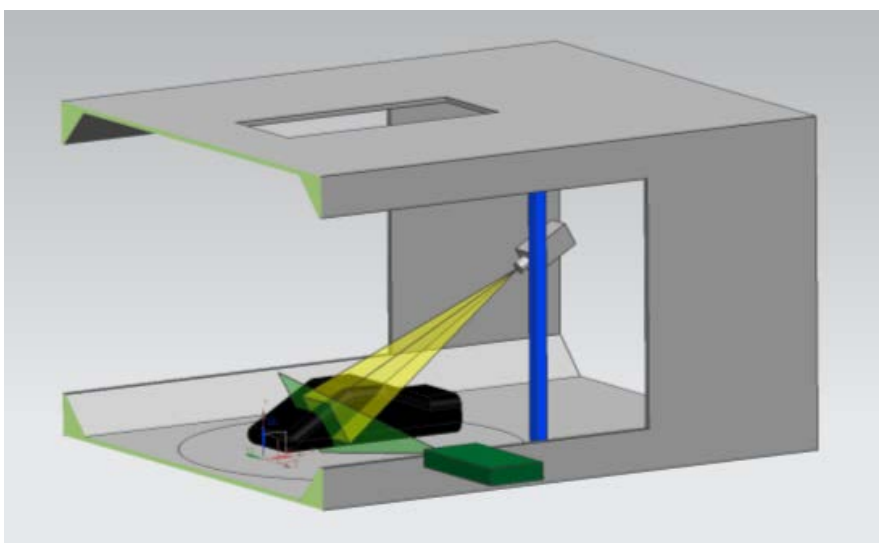

Figure 10 PIV arrangement for A pillar - Single camera- 2 component planar velocity field.

Time-averaged results are shown in Figure 10 presented as inplane streamlines upon contours of normalized (to free-stream) in-plane velocity.

Results show the A-Pillar flow structure to undergo little variation with yaw as anticipated on a radii designed to minimize changes in separation point with yaw. At $0^{\circ}$ and $-10^{\circ}$ there is no change to the flow structure with flow remaining fully attached along the A-Pillars length as it migrates from the front slant to the side. However, local velocity is greater in the $-10^{\circ}$ case due to increased acceleration around the radius as the larger yaw angle effectively reduces its curvature. 
At $-20^{\circ}$ the flow structure changes subtly toward the upper end of the A-pillar, visible in the $70 \%$ and $90 \%$ planes. Here flow separates at the radii's trailing edge and forms a small separation bubble which becomes larger with distance along the A Pillar. The structure in the lower plane is, however, consistent with those at $0^{\circ}$ and $-10^{\circ}$ showing separation to initiate at the top of the A Pillar and extend down as yaw angle increases.

At $-30^{\circ}$ separation occurs along the full extent of the A-Pillar and is now present in the lowest measurement plane. The formation of this separation bubble is driven by the increasingly adverse pressure gradient present around the radius as flow moves from an area of high acceleration/velocity and low static pressure to higher static pressure along the model side where it decelerates back to free-stream velocities. This pressure gradient becomes increasingly adverse as yaw angle increases, effectively reducing the curvature and increasing acceleration.

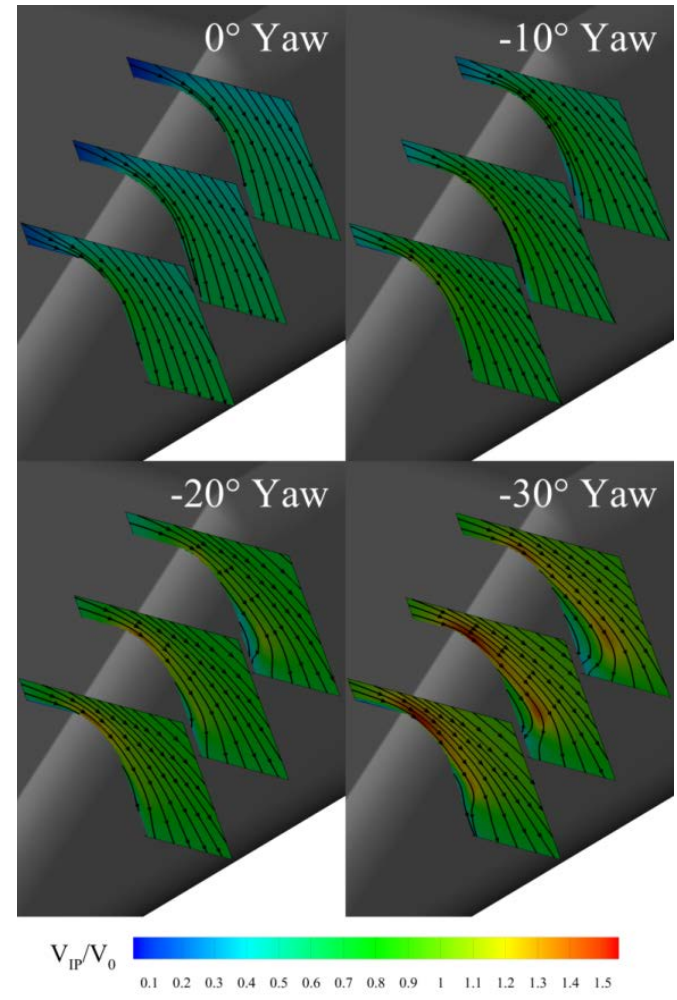

Figure $11 \mathrm{~A}$ pillar time averaged in-plane streamlines overlaid upon contours of normalized in plane velocity.

\section{Rear Notch}

A preliminary investigation using surface flow visualization methods (not presented here) showed a flow-field typical of that seen on many notchback geometries. Flow across the backlight dominated by a pair of large trailing pillar vortices which grow and encroach inboard as they form along its edges. These vortices subsequently impinge heavily on the boot-deck where there is a degree of asymmetry. This flow visualization was used to inform the location of the subsequent PIV measurements. A example configuration of the system for measurements in the notch is illustrated in Figure 11. A number of other camera orientations were employed to complete the measurements in this region.

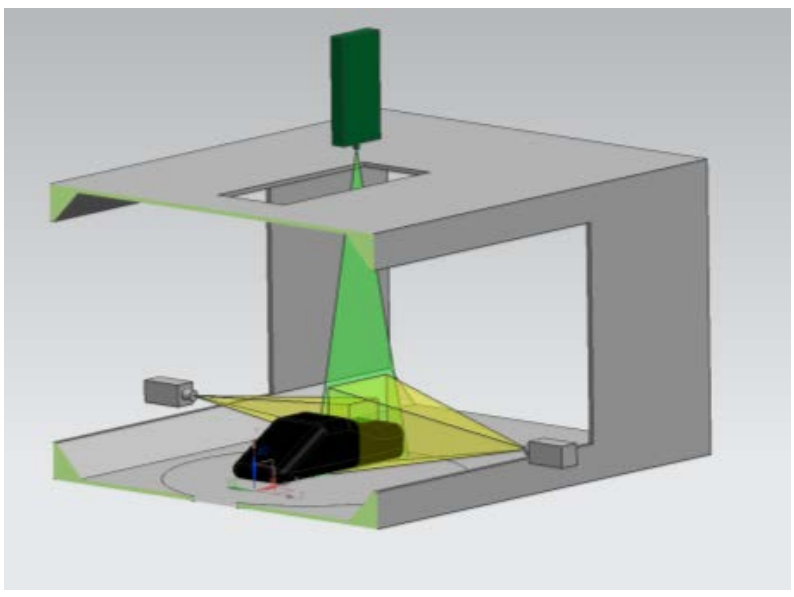

Figure 12. In-notch PIV setup. Two cameras giving an embedded high resolution area within a larger field of view.

The asymmetry present in the in-notch flow appears to be driven by a disparity in the strength of the trailing pillar vortices. This can be seen in Figure 12 where surfaces of longitudinal vorticity have been drawn through volumetric data generated from the seven time-averaged stereoscopic PIV measurements. These are presented at $\pm 35 \%, \pm 55 \%$ and $\pm 75 \%$ of normalized longitudinal vorticity and clearly show the development and strengthening of the vortices along the edge of the backlight and boot-deck. The vortex developing on the right is, however, stronger and more dominant than that its counterpart on the left, normalized vorticity peaking at -1 here compared to 0.85 , showing strength to differ by $\sim 15 \%$ across the model, a large disparity when considering these measurements to have been taken at $0^{\circ}$ yaw.

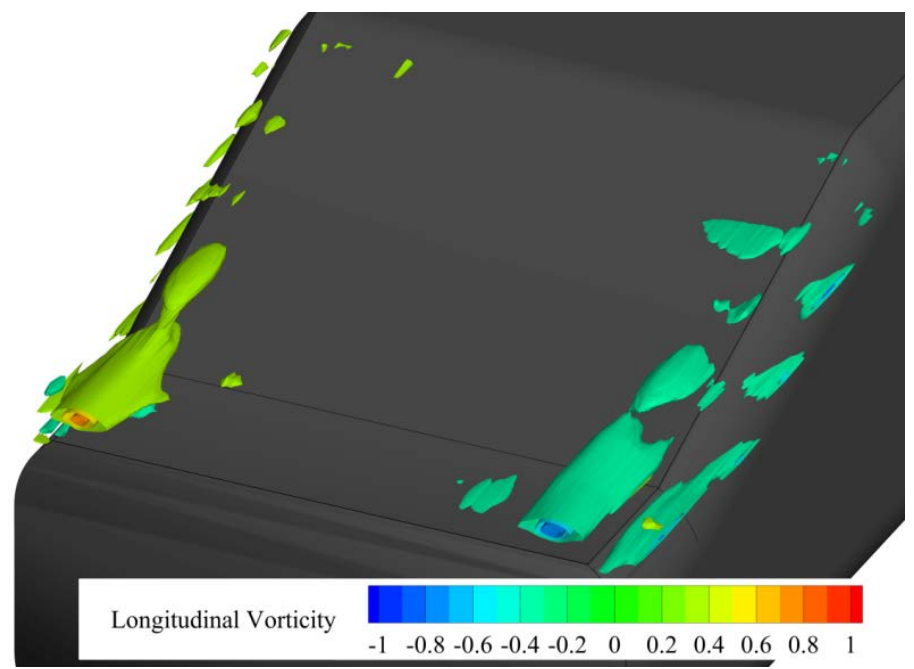

Figure 13. Surfaces of normalised longitudinal vorticity from timeaveraged stereoscopic PIV data.

Whilst the differing strength of the trailing pillar vortices is shown clearly by the stereoscopic measurements the behavior of the central region is better observed in 2D streamwise 
measurements. These are presented in Figure 13, the timeaveraged results represented by streamlines drawn upon contours of normalized in-plane velocity, for clarity these are displayed at $1 / 3^{\text {rd }}$ lateral resolution. Flow across the majority of this region can be seen to remain attached as it decelerates from a velocity peak at the backlight header. As it diffuses into the notch its deceleration is combined with a thickening of the boundary layer as the velocity deficit close to the surface increases as the flow overcomes the adverse longitudinal pressure gradient. This is most prevalent on the centerline and becomes less so toward the outer edges where the surface flow is energized by supplementary flow entrained laterally into the notch by the trailing pillar vortices.

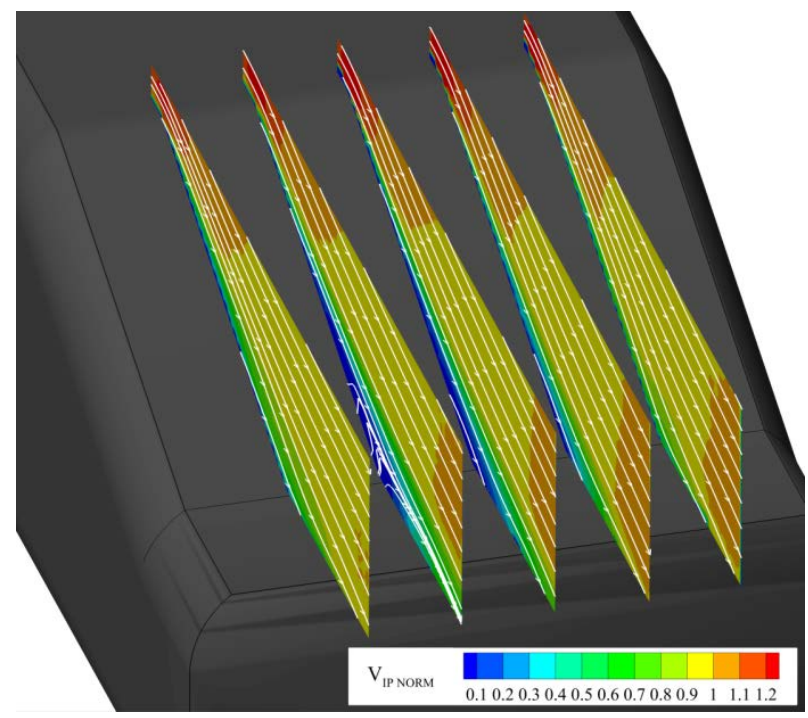

Figure 14. Streamwise 2D PIV data displayed at $1 / 3^{\text {rd }}$ lateral resolution.

The separation present in the second plane left of the centerline in Figure 13 is shown in greater detail in Figure 14 where the stream-wise planes in which it is present $(-15 \mathrm{~mm}$, $30 \mathrm{~mm},-45 \mathrm{~mm}$ and $-60 \mathrm{~mm}$ ) are shown; streamlines once again drawn upon contours of normalized in-plane velocity. The attached flow on the right of the backlight is forced significantly toward the left as it approaches the boot-deck due to the dominant trailing pillar vortices attaching here. As this moves across the base of the backlight it interacts with the flow moving down the left hand side and begins to induce separation at $\mathrm{y}=-15 \mathrm{~mm}$, which remains present up to $\mathrm{y}=$ $60 \mathrm{~mm}$. The weak nature of this separated flow can be seen in the lack of any distinct coherent structures forming within the separation bubble. Instead the time-average form shows flow forced from the surface and exiting the notch along the line of the coalesced streamlines displaced above the boot-deck surface.

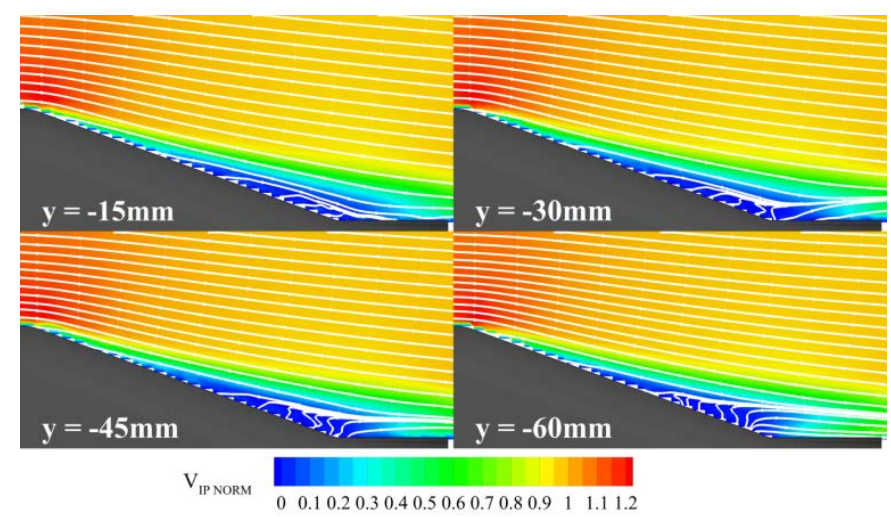

Figure 15 Streamwise 2D PIV data showing the central region of separated flow.

The interaction of this separated region of flow with that on the boot-deck is further explored in Figure 15 which shows surfaces of lateral velocity, red and blue, drawn at $\pm 20 \%$ of the maximum through the 7 stereoscopic time-averaged PIV planes. These surfaces highlight the extent of the flow entrained laterally into the notch and illustrate the dominance of the entrained flow reattaching on the boot-deck. The unbalanced nature of these flows due the imbalanced trailing pillar vortices is again evident with the right (blue) encroaching further inboard than the left (red). Toward the outer edges of the model the trailing pillar vortices are also shown, highlighted with 3D streamlines colored by normalized velocity. The white streamlines in the center are drawn along the 2D streamwise PIV planes from Figure 14 and reveal the extent of the central separated flow. It is clear from this figure that the coalesced streamlines along which the separated flow exits the notch are forced away from the boot-deck surface by the attached flow encroaching laterally toward the centerline (red). This displacement, greatest at the outboard edge where the laterally encroaching flow is thicker due to it having had less chance to diverge from the reattachment point.

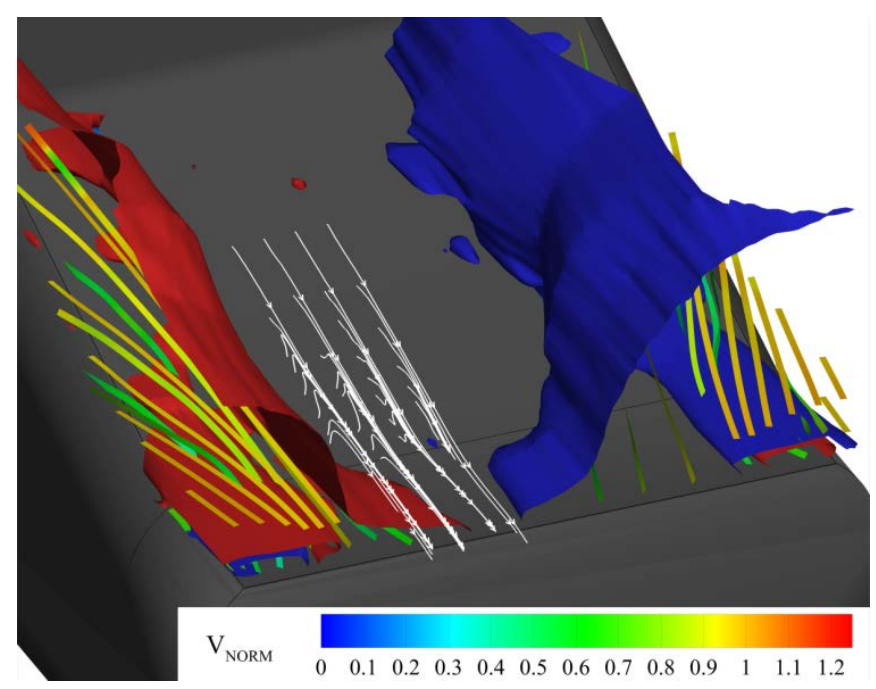

Figure 16. Combined stereoscopic and 2D PIV throughout the notch.

A weak time-averaged rotation of flow about the models longitudinal axis can be observed in Figure 16 where the $y=-$ $60 \mathrm{~mm}$ and $-45 \mathrm{~mm}$ 2D PIV planes are shown on the left and 
right, streamlines drawn upon contours of normalized in-plane velocity. Presented alongside these is the heavily cropped span wise stereoscopic plane from the backlight to boot-deck intersection, in-plane streamlines drawn upon contours of normalized longitudinal vorticity in this case. Streamlines along the span wise plane reveal a rotation of flow at the base of the separated region; a behavior induced by the angular momentum arising from the movement of flow across the base of the backlight. This structure is, however, weak and the vorticity is several orders of magnitude less than that in the trailing pillars. It is therefore not capable of persisting within the separated flow as it exits the notch and as such is not present in the stereoscopic measurement planes downstream of this point.
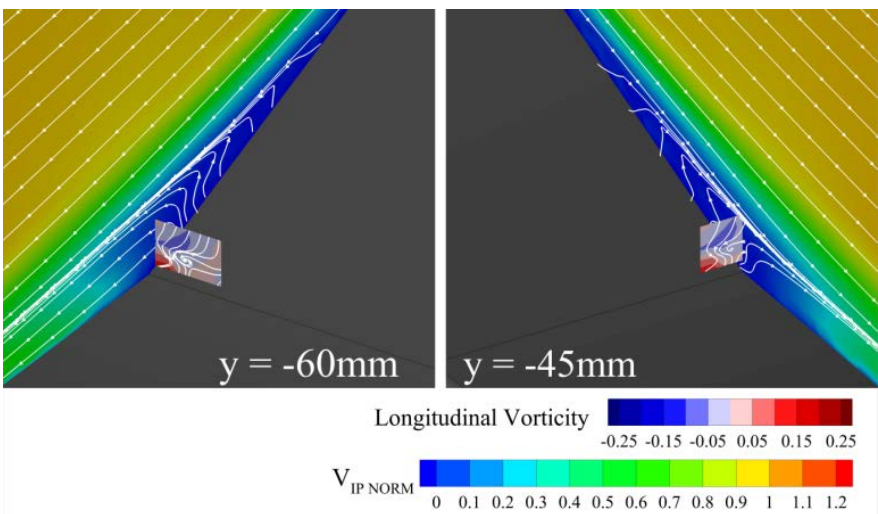

Figure 17 Combined stereoscopic and 2D PIV measurements detailing separated flow structure.

Time-averaged surface pressure measurements for the backlight, boot-deck and base are shown in Figure 17. It shows there to be a strong adverse pressure gradient along the notch that is responsible for the region of separated flow in the center. This gradient is formed as pressure recovers from a minimum at the backlight header, where flow accelerates around the radius and expands into the notch. The weak nature of the separated flow in the center of the backlight seen in the PIV is confirmed as the pressure recovery continues along its length. The presence of this separated flow is only really seen in the centerline pressure distribution (Figure 5), where a slight inflection in the pressure recovery in the middle of the slant can be seen as separation begins. Toward the outboard edge of the notch this pressure recovery is suppressed by the proximity of the trailing pillar vortex to the surface as a result of the low pressure at its core and high surface velocity induced by its rotation.

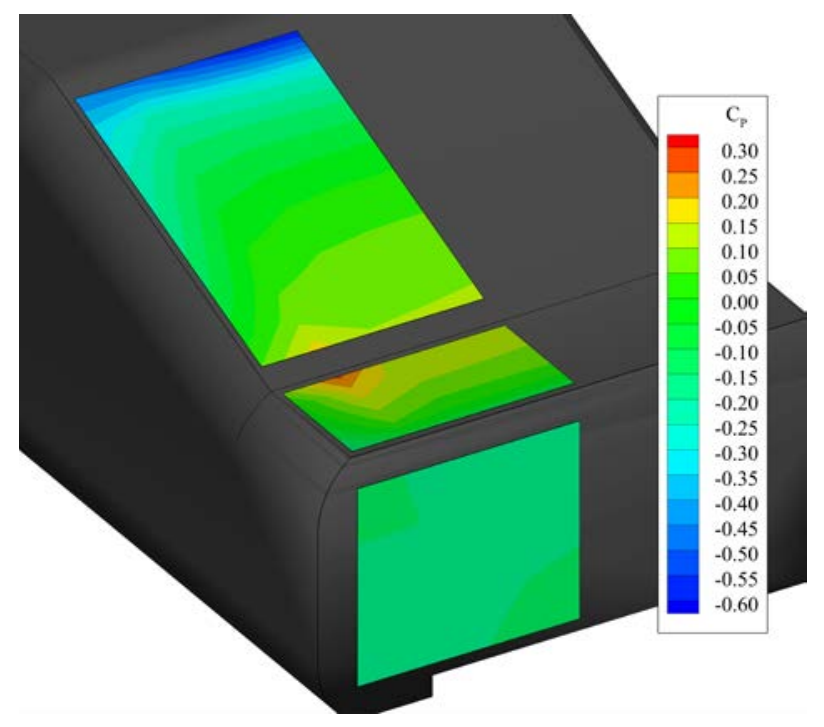

Figure 18. Time-averaged backlight, boot-deck and base pressure distribution.

The temporal behavior of surface pressure is shown in Figure 18 , the RMS magnitude throughout the notch and across the base shows the surface pressure to be largely stable. This is particularly evident at the outboard edge of the backlight where fluctuation levels approach a minimum along the trailing pillar vortex reattachment line. Inboard of this, in the upper half of the backlight where the attached flow diffuses into the notch, fluctuation intensities remain low due to the inherent stability of this type of flow. However, as this propagates downstream and separates across the lower half of the backlight, fluctuations increase. Whilst the weak nature of this separation has little effect upon time-averaged pressure its instability, arising from the benign manner in which it separates, does lead to these higher fluctuation levels which clearly pick out the extent of the separated flow.

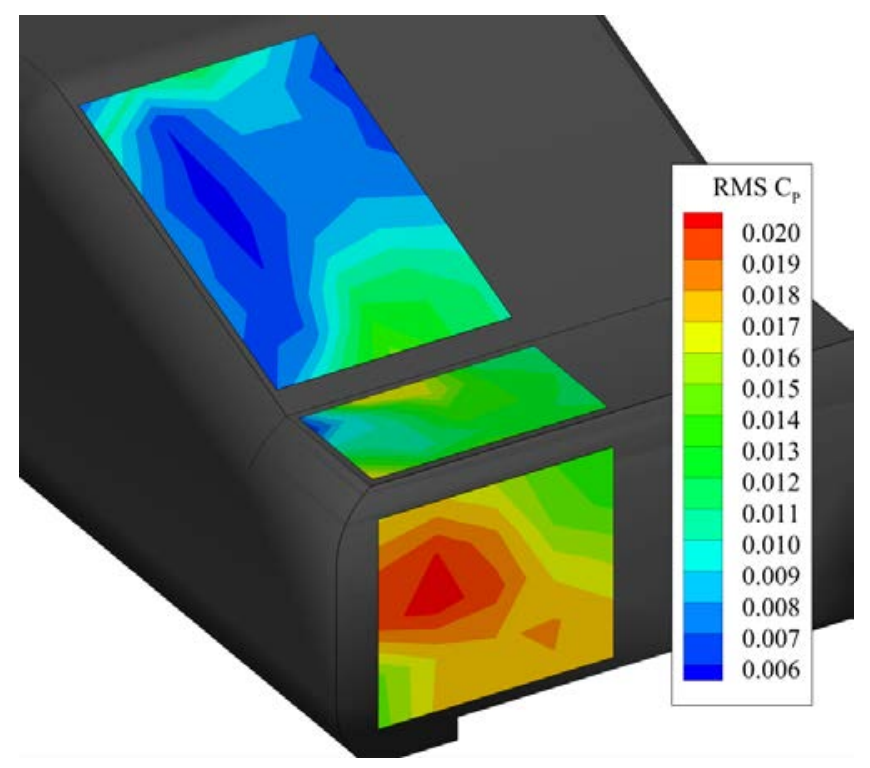

Figure 19 RMS magnitude of surface pressure fluctuations throughout the notch and across the base. 
Instability is typically higher across much of the boot-deck due to the separated shear layer impinging strongly upon this surface, coupled with unsteadiness induced by the strong interaction of the two opposing laterally entrained flows. This unsteadiness is also potentially exacerbated by flow from the separated region propagating downstream above this surface further manipulating the local pressure field. Peak RMS magnitude occurs at a location coincident with the timeaveraged pressure peak showing there to be a strong correlation between impingement strength and small scale surface pressure instabilities.

RMS velocity fluctuations of the off surface flow above the backlight along the 5 stereoscopic PIV planes, Figure 19, reveal much the same behavior. Close to the backlight header unsteadiness is restricted to the thin boundary layer, arising from its turbulent nature and the high levels of shear. These fluctuations, however, do not manifest themselves in surface pressure due to their small scale highly localized effects. As flow propagates through the notch an area of elevated unsteadiness forms in the upper half of the backlight directly upstream of the time-averaged separation, circled in red. Indicating the separated region to occasionally extend as far upstream as these planes, behavior not clear in surface pressure fluctuations due to the weak pressure field associated with it. Downstream, instability is high within the time-averaged extent of the separated flow due to its inherently unstable nature. Also visible are the two regions of extreme unsteadiness toward the outer edges of the boot-deck, circled white, induced by the strong impingement of the reattaching flow here which produces significant velocity and pressure fluctuations.

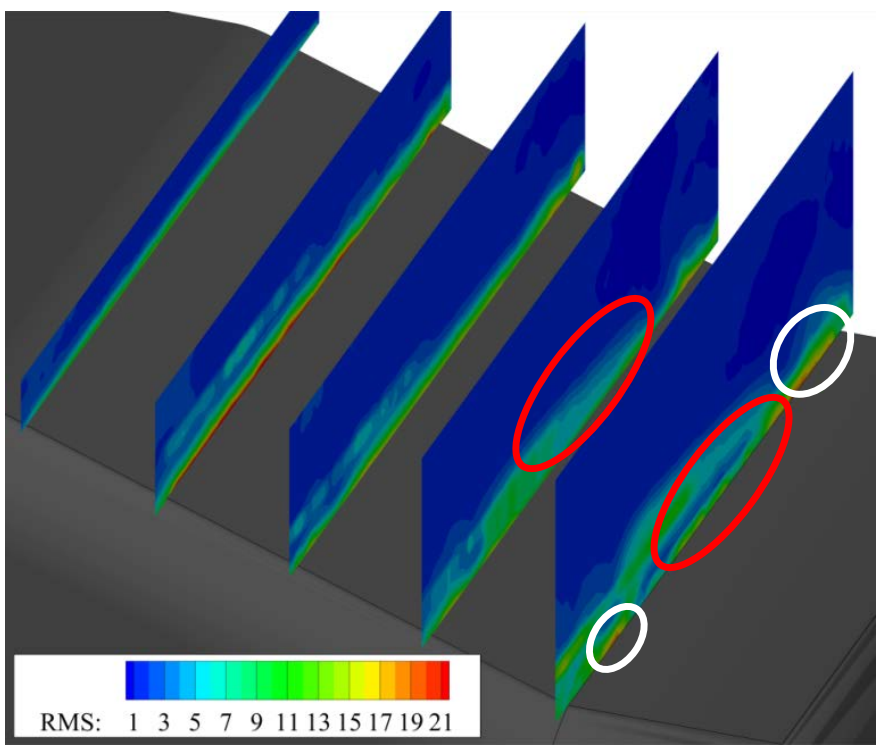

Figure 20 RMS magnitude of velocity fluctuations above the slant.

RMS velocity fluctuations above the boot-deck are shown in Figure 20 and continue to reveal high levels of unsteadiness within the region identified in the time-averaged data as separated flow. This highly erratic flow can be seen to induce significant instability within the flow beneath it which diverges laterally across the boot-deck toward the centerline. The extent of the unsteadiness induced by the separated flow is clear when comparing this region to its counterpart on the right where fluctuations are minimal; this implies RMS pressure to be asymmetric and significantly reduced on the right across the unmeasured region. Along the most rearward plane, shown on the right in Figure 20, fluctuations peak across a thin layer immediately above the surface due to the proximity of this flow to the small scale instabilities in the shear layer separating from the boot-deck trailing edge.

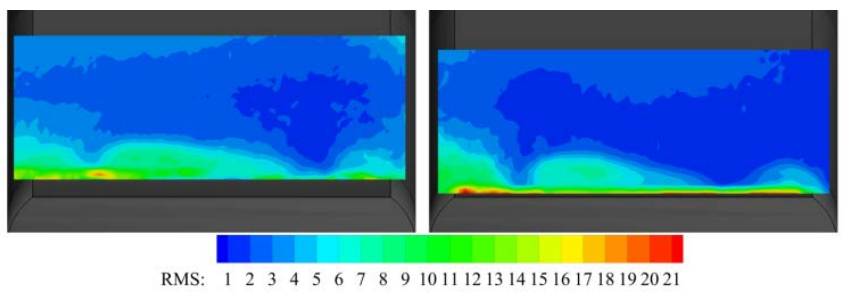

Figure 21. RMS magnitude of velocity fluctuations above the bootdeck.

\section{Summary/Conclusions}

1. The availability of high quality experimental data for validation of computational codes is essential if progress is to be made in developing simulation capability.

2. The SAE 20 degree backlight angle notchback reference model exhibits the important flow features and flow physics of a road vehicle while avoiding unnecessary additional modeling and difficulty of interpretation. It is an ideal test case for numerical validation.

3. Experimental data for the impingement region, model centerline, A pillar and multiple planes on the slant and boot-deck of the reference model are presented.

4. The impingement zone exhibits attached flow with increasing cross plane velocities at the model edges.

5. The 'A' pillar data demonstrates an attached flow apart from at the higher yaw angles where regions of separation become evident, particularly towards the top of the A pillar.

6. The highly $3 \mathrm{D}$ flow in the notch is captured in considerable detail and shows a typical notch back flow structure with asymmetry in the trailing vortex structure and in the impingement on the boot-deck. Weak separation on the slant with flow displaced above the boot-deck is highlighted and the correlation between the PIV results and the surface pressures is demonstrated.

7. Example data is included throughout the paper and full data sets including $x, y, z, V x, V y, V z$ and $\operatorname{RMS}(x, y, z)$ and full model CAD are available in the Loughborough University Institutional Repository as a resource for future code development [1].

\section{References}

1. Passmore, M.A. Perry A.K., Wood, D.W. SAE reference model - 20 degree notchback validation dataset (reference SAE paper 2014-01-0590) at: https://dspace.lboro.ac.uk/dspacejspui/handle/2134/13886

2. Morel T. Aerodynamic drag of bluff body shapes characteristic of hatch-back cars. SAE Paper 780287 (1978) 
3. Ahmed, SR, Ramm, G, Faltin, G. Some salient features of the time-averaged ground vehicle wake. SAE paper 840300 (1984)

4. Le Good G, Garry KP, On the use of reference models in automotive Aerodynamics. SAE Paper 2004-01-1308

5. Heft, A, Indinger, T, Adams, NA. Introduction of a new realistic generic car model for aerodynamic investigations. SAE Paper 2012-01-0168 (2012)

6. Johl, G., Passmore, M.A., Render, P.M., Design methodology and performance of an in-draft wind tunnel. The Aeronautical journal, Vol. 108, pp115-124. (2004)

7. SAE International. Aerodynamic Testing of Road Vehicles - Open Throat Wind Tunnel Adjustment. J2071 (1990)

8. Cogotti, A. A Parametric Study on the Ground Effect of a Simplified Car Model. SAE paper 980031 (1998)

9. Ishima, T., Takahashi, Y., Okado, H., Baba, Y. et al. 3DPIV Measurement and Visualization of Streamlines Around a Standard SAE Vehicle Model. SAE paper 201101-0161 (2011)

10. Schröck, D., Widdecke, N., Wiedemann, J. Aerodynamic Response of a Vehicle Model to Turbulent Wind. FKFS (2010)

11. Sims-Williams DB, Dominy RG, Experimental Investigations into the unsteadiness and instability in passenger car aerodynamics. SAE Technical paper series, 980391, World Congress, Detroit, 1998

12. Westerweel J, Fundamentals of digital particle image Velocimetry, Measurement Science and Technology, Vol.8, pp.1379-1392 (1997)

13. Passmore M A, Spencer A, Jowsey, L, Newnham, $P$, Wood DW, The application of Particle Image Velocimetry in automotive aerodynamics. SAE paper 2010-01-0120 (2010)

14. SAE International. Aerodynamic Testing of Road Vehicles - Closed-Test-Section Wind Tunnel Boundary Interference. J2085 (1991)

\section{Contact Information}

Martin Passmore : $\underline{\text { m.a.passmore@lboro.ac.uk }}$

The full data sets can be accessed from the Loughborough University institutional repository at:

https://dspace.lboro.ac.uk/dspaceispui/handle/2134/13886

Included in the download are details of the model geometry, the time averaged and RMS PIV data, the surface pressure data and the force and moment data.

\section{Acknowledgments}

The authors would like to acknowledge the contributions made by Andrew Mansfield and Matt Passenger who assisted in the acquisition of some data sets. Thanks are also due to Rob Hunter for his tireless work keeping the wind tunnel facility running smoothly and building models for test. 